\title{
Treatment of radiation-induced cystitis and vulvodynia via a ganglion impar block using a lateral approach under computed tomography guidance -a case report-
}

\author{
Jeong-Eun Lee, Kyung-Hwa Kwak, Seong Wook Hong, Hoon Jung, \\ Seung-Yeon Chung, and Jun-Mo Park \\ Department of Anesthesiology and Pain Medicine, Kyungpook National University School of Medicine, Daegu, Korea
}

\begin{abstract}
Adjuvant radiation therapy (RT) after colorectal cancer surgery can prevent local recurrence, but has several side effects. Precise injection of drugs into the affected areas is complicated by radiation-induced fibrosis of soft or connective tissue. A 48-year-old woman experienced severe intractable perineal pain, dysuria, urinary urgency, and frequent urination after rectal cancer surgery and adjuvant RT, and was diagnosed with radiation-induced cystitis and vulvodynia. Her symptoms persisted despite two fluoroscopy-guided ganglion impar blocks. Fluoroscopy revealed atypical needle tip positioning and radiolucent dye distribution, presumably due to radiation-induced fibrosis in the target region. We performed two computed tomography (CT)-guided ganglion impar blocks by using a lateral approach, which allowed more accurate positioning of the needle tip. Her pain visual analog score decreased from 9 to 3 , and she recently resumed sexual intimacy. $\mathrm{CT}$ guidance is a viable alternative to fluoroscopy guidance when performing ganglion impar blocks in fibrotic areas.
\end{abstract}

Key Words: Cystitis, Nerve block, Radiation, Radiotherapy, Sympathetic ganglia, Vulvodynia.

The incidence of colorectal cancer has recently escalated in Korea due to an increasingly westernized diet as well as other causes [1]. Although advances in diagnosis and treatment have

Corresponding author: Jun-Mo Park, M.D., Ph.D.

Department of Anesthesiology and Pain Medicine, Kyungpook National University School of Medicine, 130, Dongdeok-ro, Jung-gu, Daegu 41944, Korea

Tel: 82-53-420-5863, Fax: 82-53-426-2760

Email:pjm4013@naver.com

ORCID: http://orcid.org/0000-0001-9234-5177

Received: July 6, 2016.

Revised: August 31, 2016 (1st); September 20, 2016 (2nd).

Accepted: September 21, 2016.

Korean J Anesthesiol 2017 February 70(1): 81-85

https://doi.org/10.4097/kjae.2017.70.1.81 improved the survival rate of colorectal cancer patients [2], the number of colorectal cancer survivors suffering from treatmentrelated complications has not declined [3]. Adjuvant radiation therapy (RT) is currently one of the major therapeutic methods for preventing local recurrence of colorectal cancer before or after surgery [4]. However, despite its many advantages, it has unavoidable complications, such as radiation cystitis and vulvodynia, which are caused by unintentional exposure of the bladder and perineal region to radiation during adjuvant RT. These are both major complications, which reduce quality of life and can potentially cause severe pain [5], and have no established treatment.

It is extremely difficult to inject drugs accurately into the affected area, particularly when radiation-induced fibrosis of the soft or connective tissue has occurred [6]. Here, we report the

(c) This is an open-access article distributed under the terms of the Creative Commons Attribution Non-Commercial License (http://creativecommons.org/ licenses/by-nc/4.0/), which permits unrestricted non-commercial use, distribution, and reproduction in any medium, provided the original work is properly cited. 
case of a patient who complained of severe intractable perineal pain, dysuria, and urinary frequency and urgency, which were caused by administration of adjuvant RT after rectal cancer surgery. Due to post-RT fibrosis, ganglion impar blocks administered under fluoroscopic guidance were unsuccessful. However, when a ganglion impar block was administered using a lateral approach under computed tomography (CT)-guidance, the block was successful.

\section{Case Report}

A 48-year-old female patient was referred to us from a gynecologic department for severe intractable perineal pain. She had undergone rectal cancer surgery 18 months prior to visiting our pain clinic, after which she had received adjuvant RT 20 times during a 2-month period. She complained of severe intractable perineal pain, dysuria, and urinary urgency and frequency, which had persisted after adjuvant RT. Only a brief mention was made to her that her symptoms were side effects of the RT, rather than due to specific abnormalities, whereas she underwent all required examinations in the departments of urology and gynecology before visiting our pain clinic. Before her visit, she had been admitted to the emergency room three times for severe intractable perineal pain. At the pain clinic, she complained of severe intractable perineal and perivaginal pain, with pricking, stabbing, and throbbing sensations, and her pain visual analog scale (VAS) score varied from 6 to 9 out of 10 . The pain usually occurred spontaneously and persisted all day, and it was aggravated by urination. Although we did not assess the altered pin-prick threshold because of her intense anxiety and reluctance to undergo the examination, her total score on the Leeds Assessment of Neuropathic Symptoms and Signs (LANSS) pain scale was 15 [7].

We considered that neuropathic events probably contributed to her pain, and we fully explained our treatment plan to her. We intended to perform a ganglion impar block three times per week; if the outcomes of the first two blocks were successful, alcohol neurolysis would be considered. The blocks were firstly administered using a fluoroscopy-guided trans-sacrococcygeal approach, without complications. However, unusually, the spinal needle tips were found to be located notably more deeply from the anterior bony surface line than are typically observed (Fig. $1 \mathrm{~A})$, and the spreading patterns of the radiolucent contrast dye were atypical (Fig. 1B). After the first block, the patient's VAS score changed minimally, although the urinary symptoms and pain were somewhat reduced. After the second block, the patient complained of pain aggravation, and did not return to the pain clinic for 1 year. During that time, she only took $300 \mathrm{mg}$ pregabalin and $20 \mathrm{mg}$ oxycodone daily, which had been prescribed by physicians in the departments of gynecology and urology.

After 1 year, her gynecologist recommended another visit to the pain clinic. Upon her return, her VAS score and degree of pain were unchanged, and the symptoms of radiation cystitis and vulvodynia due to adjuvant RT were also persisting. She had been admitted to the emergency room several times in the interim due to episodes of severe intractable perineal pain. A ganglion impar block was again performed, but the fluoroscopic images were not significantly different from those observed previously, and again the outcome of the procedure was not successful. We believed treatment failure was due to injection at the

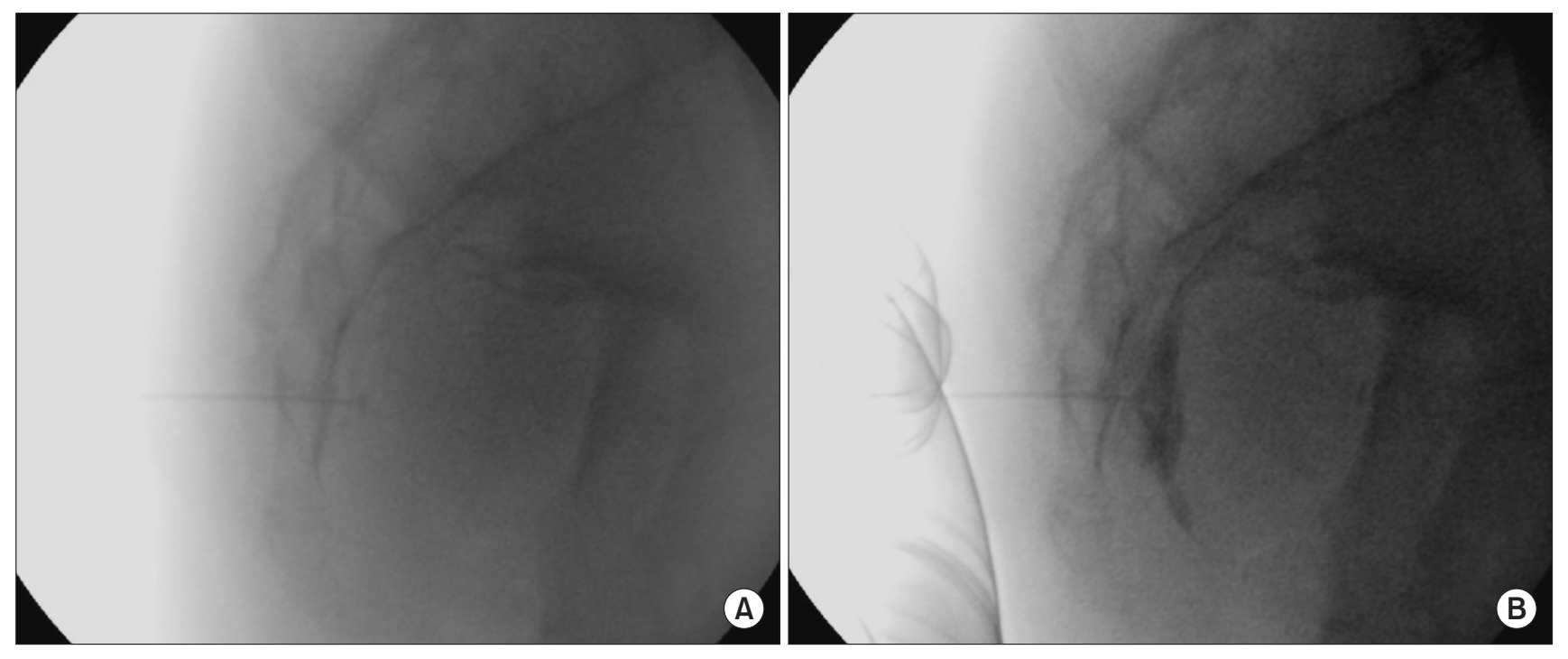

Fig. 1. Fluoroscopic images of administration of a ganglion impar block via a trans-sacrococcygeal approach. (A) The spinal needle tip is located deeper to the anterior bony surface line than is typically observed. (B) The spreading pattern of the radiolucent contrast dye is atypical. 
wrong site. Owing to the radiation-induced fibrosis of soft or connective tissue around the target site, it was extremely difficult to inject the target site precisely. Hence, we decided to perform a ganglion impar block under CT-guidance, which would allow us to insert a Chiba needle into the sacrococcygeal junction at the anterior surface line.

The patient was connected to a peripheral IV line and placed in the prone position on a CT table under monitored anesthesia. Metal clip markers were placed on her buttocks to serve as landmarks for identifying the puncture site. The region from the upper endplate of the S3 vertebra to the tip of the coccyx was scanned to identify a suitable skin entry point for the appropriate approach to the target region. Based on an axial CT fluoroscopy (CTF) image, the target region and the skin entry point were selected at a level parallel to the most anterior portion of the sacrococcygeal junction. The skin entry point was marked $12 \mathrm{~cm}$ lateral to the midline on the right and was prepared with an antiseptic solution. Local anesthesia (2\% lidocaine) was administered. A 22-gauge Chiba needle (needle length: $15 \mathrm{~cm}$ ) was then advanced trans-axially and medially toward the anterior surface of the sacrococcygeal junction under intermittent CTF guidance. After confirming contact with the lateral edge of the coccyx, the Chiba needle was pushed along the anterior surface of the coccyx (Fig. 2A). Once it was verified that the tip of the Chiba needle was correctly positioned, $0.5 \mathrm{ml}$ of a radiolucent contrast dye was injected four times, as we wanted to confirm whether the dye could fully cover the target lesion (Fig. 2B). Sufficient spreading of the dye around the target region was confirmed by CT scan (Fig. 2C). We then injected a mixture containing $6 \mathrm{ml}$ of $0.2 \%$ ropivacaine, $3 \mathrm{ml}$ of $0.5 \%$ lidocaine, 20 $\mathrm{mg}$ of triamcinolone, and $6000 \mathrm{IU}$ of hyaluronidase. We firstly attempted the injection using a $10 \mathrm{ml}$ syringe, as is typically used in such cases, but the resistance to the injection was too strong, unlike when the procedure was performed under fluoroscopic guidance. We therefore divided the mixture into $2 \mathrm{ml}$ syringes; although the resistance was somewhat less, it was still quite strong.

The patient's VAS score had decreased from 9 to 6 (out of 10) by 1 week after the ganglion impar block. However, unlike with the unsuccessful outcome of the earlier fluoroscopy-guided procedure, the outcome of the CT-guided procedure was satisfactory to the patient. The procedure was repeated after 1 week, and the injection resistance was significantly reduced. The patient's VAS score was less than 3 (out of 10) by 1 week after the second procedure, and she was very pleased with this outcome. Although the symptoms of radiation cystitis, including dysuria, and urinary urgency and frequency, still remained when retaining urine or urinating, the severe intractable perineal and perivaginal pain due to radiation-induced vulvodynia subsided markedly.

We planned to perform neurolysis with alcohol if the patient's symptoms had significantly improved after the ganglion impar blocks; however, the patient declined this treatment. We had also considered another treatment if the symptoms of radiation cystitis persisted after ganglion impar block, but the patient refused further treatment.

After her revisit, she took $150 \mathrm{mg}$ pregabalin, $30 \mathrm{mg}$ duloxetine, and $10 \mathrm{mg}$ hydromorphone daily for 3 months. Vulvodynia improved after the CT-guided block, allowing a reduction in her medication to $75 \mathrm{mg}$ pregabalin and $30 \mathrm{mg}$ duloxetine daily. After another 3 months, her VAS score remained below 3 , at which point she discontinued these medications. Thereafter,
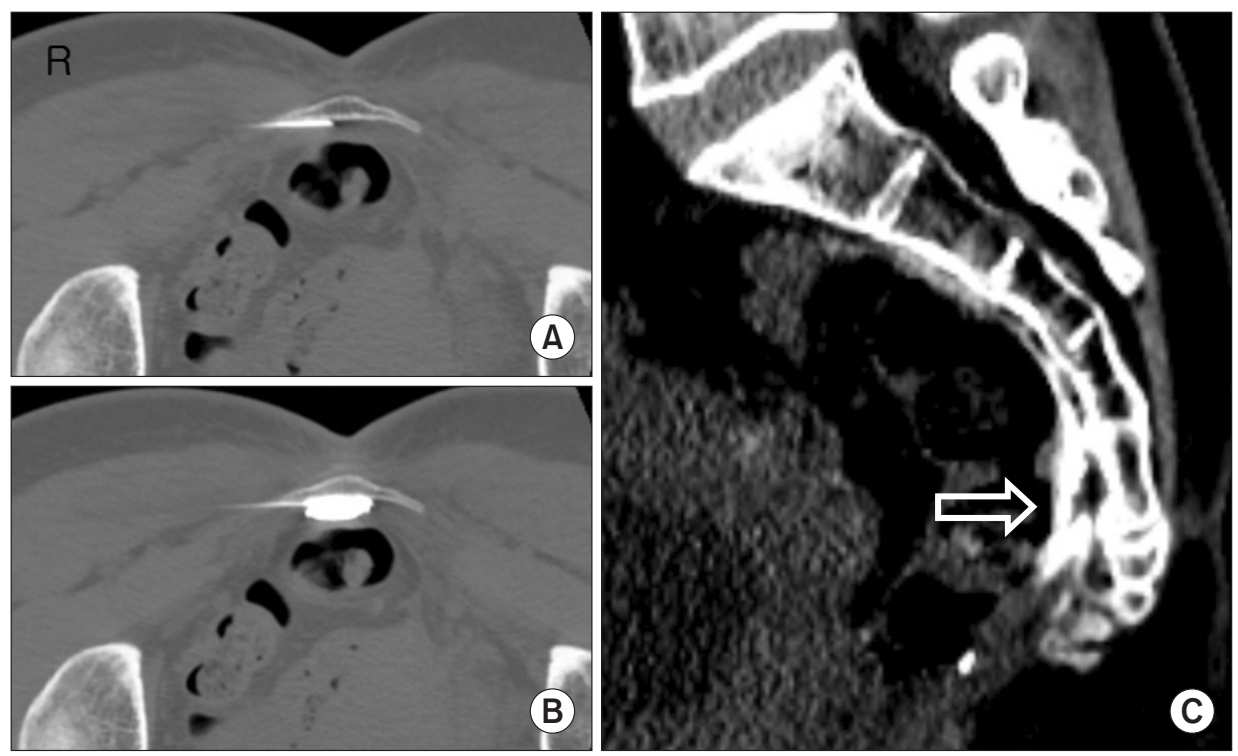

Fig. 2. Computed tomography (CT) images of administration of a ganglion impar block via a right lateral approach. (A) After confirming contact with the lateral edge of the coccyx, a Chiba needle was pushed along the anterior surface of the coccyx. (B) After confirming that the tip of the Chiba needle was correctly positioned, $0.5 \mathrm{ml}$ of radiolucent contrast dye was injected four times. (C) CT scanning showed sufficient spread of the radiolucent contrast dye around the target region. 
her symptoms did not worsen and sexual intercourse was possible. Accordingly, we recommended that she visit the pain clinic again only if necessary.

\section{Discussion}

Diarrhea, cystitis, and perineal dermatitis mainly result from the early toxicity that occurs after adjuvant RT for treatment of rectal cancer, while bowel dysfunction, fecal incontinence, bleeding, perforation, genitourinary dysfunction, and pelvic fractures are mainly due to the late toxicity [3]. Radiation cystitis is caused by unintentional exposure of the bladder to radiation during adjuvant RT for treatment of rectal cancer in the pelvis [8]. Acute radiation cystitis is the consequence of damage to the bladder mucosa, when the cells within the mucosa divide rapidly. This usually occurs during the healing process and is accompanied by acute inflammatory responses and tissue edema; its main symptoms are dysuria, and urinary frequency and urgency. In most cases, the course of radiation cystitis is self-limiting, rarely lasting more than 3 months after completion of the radiation treatment. Therefore, acute radiation cystitis is treated conservatively, and symptomatic relief is usually achieved by means of anticholinergic drugs.

Tissue injury after pelvic RT causes inflammation, fibrosis, and necrosis of pelvic vessels or nerves within 12-36 months. In women in particular, depending on the total dose of RT delivered to the vagina and pelvic cavity, the epithelium of the vagina and pelvic organs undergo rapid cell cycling [3]. Therefore, radiation-related vulvodynia also results in sexual symptoms, such as dyspareunia, and is associated with recurrent cystitis.

However, in the present case, the pain had lasted for more than 3 months after adjuvant RT; the course was thus not selflimiting, and conservative treatment was largely ineffective. When considering all aspects of a patient's pain, allodynia, as measured via the LANSS pain scale, rather than neuropathic events, is usually primarily responsible [7]. In our case, we believe that unintended irradiation of the otherwise normal bladder caused inflammatory pain, resulting in maladaptive reorganization in the nervous system via peripheral and central sensitization pathways. This, in turn, led to neuropathic pain via a process involving neuroplasticity and long-term potentiation of the pain, and eventually progressed to chronic pain [9].

The patient had perineal and perivaginal pain, which she described as pricking, stabbing, and throbbing, and which had lasted for more than 3 months. She was diagnosed with postoperative adjuvant RT-induced vulvodynia by a gynecologist. Commonly, cancer-related neuropathy due to radiation or chemotherapy-induced nerve damage involves neuropathic pain. The mechanism of progression from vulvodynia to neuropathic pain is similar to that by which radiation cystitis progresses to neuropathic pain [10].

Although the relationship between neuropathic pain and the sympathetic nervous system is controversial, the relationship between chronic pain and the sympathetic nervous system is well established. Sympathetic nerve blocks have been used to treat chronic pain for many years [11]. Under normal physiological conditions, the efferent sympathetic nerve and the primary afferent neurons function separately. However, after nerve injury or tissue inflammation, chemical and anatomical coupling between the sympathetic postganglionic fiber and the primary sensory neurons occurs [12]. Several mechanisms appear to be involved, such as abnormal activation of the adrenergic receptors of the primary afferents, direct interaction between efferent sympathetic fibers and primary afferent neurons during regeneration and sprouting, and possible indirect coupling involving neurovascular transmission.

The ganglion impar controls the nociceptive and sympathetic fibers of the perineum, distal rectum, perianal region, distal urethra, vulva/scrotum, and the distal third of the vagina, and also controls the sympathetic fibers of the pelvic viscera [13]. Until recently, the identity of the neural pathway associated with the ganglion impar and the indications for a ganglion impar block were unresolved. Initially, a ganglion impar block was performed to treat sympathetically mediated cancer pain in the perineum [14]. Subsequent studies have reported the effects of ganglion impar blocks on various benign or malignant diseases [13].

There are essentially four approaches to the ganglion impar under fluoroscopic guidance; the original approach is a midline technique in which a bent spinal needle is passed through the anococcygeal ligament and the needle tip is placed at the sacrococcygeal junction of the retroperitoneum. Other modified techniques are the paramedian approach through the anococcygeal ligament, the trans-coccygeal ligament approach, or the direct intercoccygeal junction approach, as well as the lateral approach [13]. In fact, the exact location of the ganglion impar is varies widely among individuals. In general, the ganglion impar lies toward the coccyx tip from the sacrococcygeal joint, and toward the lateral aspect of the sacrococcygeal joint. Hence, various methods of approach have been introduced, in addition to the above mentioned methods.

A ganglion impar block usually involves a trans-sacrococcygeal approach under fluoroscopic guidance. Fluoroscopy can reduce the risk of intravascular injection and injection of the rectum and sacrococcygeal disc, which occurs when the needle is inserted either too deep or too superficially [15]. When a radiolucent contrast dye is used during a ganglion impar block, it is easy to determine the exact location of the needle and to predict the degree to which the drug has spread, by means of fluoroscopy. However, fluoroscopy cannot identify potential ab- 
normalities in soft or connective tissue.

In the case described here, the first two fluoroscopic-guided ganglion impar blocks were ineffective, presumably because the tip of the Chiba needle was positioned much deeper than in similar cases. Precise placement of the Chiba needle in the target region was likely impeded by the fibrosis in this region, which had been caused by the postoperative adjuvant RT. In order to place the Chiba needle as close to the anterior surface of the bone as possible, which we considered would be more a more effective approach, we administered the ganglion impar block using a lateral approach under CT guidance. This has several advantages. First, it can reduce the risk of rectal perforation. The bent needle causes more injuries to the surrounding tissues and structures. Intentional bending of the needle is unnecessary under CT guidance; the needle can be positioned without perforating ossified structures [13]. Second, when using a transsacrococcygeal approach under fluoroscopic guidance, there is a substantial risk of infection because the skin entry point of the needle is closer to the anus. However, when using a lateral approach under CT guidance, there is little risk of anal-related infection if the anus is sufficiently disinfected during draping. Third, as shown in the present case, a lateral approach under intermittent axial CTF guidance allows accurate and safe placement of the needle when the target region is fibrotic.

Although there are more concerns about the radiation dose of a CT-guided technique than of a fluoroscopy-guided method, insertion of Chiba needle was less affected by RT-induced tissue fibrosis; further, the procedural time could be shortened owing to the accurate visualization facilitated by CT. Thus, an exactly administered ganglion impar block could increase the satisfaction and quality of life of the patient. Observational studies comparing the radiation dose and therapeutic effect between fluoroscopy- and CT-guided techniques should be performed in future.

As mentioned above, various approaches for performing ganglion impar block have been introduced, and CT-guided technique has already been presented as an alternative means of guidance. There has been no previous report of a case with these specific indications, in which a CT-guided technique was required despite the many concerns regarding the total radiation dose. When it is impossible to inject a drug accurately into the target region due to pre- or postoperative adjuvant RT-induced fibrosis, we suggest that the CT-guided technique should be used without hesitance. Hence, this case is significant in demonstrating a practical alternative for inaccurate ganglion impar block.

In conclusion, this case convincingly demonstrates that a CTguided lateral approach is a viable alternative to a fluoroscopyguided trans-sacrococcygeal approach when performing ganglion impar blocks in areas affected by adjuvant RT-induced fibrosis.

\section{References}

1. Jung KW, Won YJ, Kong HJ, Oh CM, Seo HG, Lee JS. Cancer statistics in Korea: incidence, mortality, survival and prevalence in 2010. Cancer Res Treat 2013; 45: 1-14.

2. Park HC, Shin A, Kim BW, Jung KW, Won YJ, Oh JH, et al. Data on the characteristics and the survival of korean patients with colorectal cancer from the Korea central cancer registry. Ann Coloproctol 2013; 29: 144-9.

3. Joye I, Haustermans K. Early and late toxicity of radiotherapy for rectal cancer. Recent Results Cancer Res 2014; 203: 189-201.

4. Cohen AM. Adjuvant therapy in rectal cancer. Hepatogastroenterology 1992; 39: 215-21.

5. Payne H, Adamson A, Bahl A, Borwell J, Dodds D, Heath C, et al. Chemical- and radiation-induced haemorrhagic cystitis: current treatments and challenges. BJU Int 2013; 112: 885-97.

6. Gieringer M, Gosepath J, Naim R. Radiotherapy and wound healing: principles, management and prospects (review). Oncol Rep 2011; 26: 299-307.

7. Bennett M. The LANSS Pain Scale: the Leeds assessment of neuropathic symptoms and signs. Pain 2001; 92: 147-57.

8. Smit SG, Heyns CF. Management of radiation cystitis. Nat Rev Urol 2010; 7: 206-14.

9. Voscopoulos C, Lema M. When does acute pain become chronic? Br J Anaesth 2010; 105 Suppl 1: i69-85.

10. Danby CS, Margesson LJ. Approach to the diagnosis and treatment of vulvar pain. Dermatol Ther 2010; 23: 485-504.

11. Wu CL, Marsh A, Dworkin RH. The role of sympathetic nerve blocks in herpes zoster and postherpetic neuralgia. Pain 2000; 87: 121-9.

12. Jänig W, Levine JD, Michaelis M. Interactions of sympathetic and primary afferent neurons following nerve injury and tissue trauma. Prog Brain Res 1996; 113: 161-84.

13. Scott-Warren JT, Hill V, Rajasekaran A. Ganglion impar blockade: a review. Curr Pain Headache Rep 2013; 17: 306.

14. de Leon-Casasola OA. Superior hypogastric plexus block and ganglion impar neurolysis for pain associated with cancer. Tech Reg Anesth Pain Manag 1997; 1: 27-31.

15. Wemm K Jr, Saberski L. Modified approach to block the ganglion impar (ganglion of Walther). Reg Anesth 1995; 20 : 544-5. 